\title{
1 Title: Slower organisms exhibit sudden population 2 disappearances in a reddened world
}

\section{Authors:}

4 Christopher J. Greyson-Gaito ${ }^{\star 1}$, Gabriel Gellner ${ }^{1}$, Kevin S. McCann ${ }^{1}$

\section{Affiliations:}

6 1. Department of Integrative Biology, University of Guelph, Guelph, Ontario, Canada

$7 \quad{ }^{*}$ Corresponding Author - Email: christopher@greyson-gaito.com (CJGG)

\section{ORCID:}

9 CJGG - 0000-0001-8716-0290

10 GG - 0000-0001-8170-1463

11 KSM - 000-0001-6031-7913 


\section{Abstract}

13 Sudden population disappearances are a possibility in natural systems. Human-caused

14 reddening of noise - increasing the autocorrelation of noise - may make sudden population

15 disappearances more likely because red noise can enlarge the period and magnitude of

16 perturbations, potentially overwhelming species' natural compensatory responses and

17 pushing species' population dynamics away from equilibria into transient dynamics and close

18 to extinction. Furthermore, species' natural compensatory responses are related to life history

19 along the slow-fast continuum. This interaction of slow-fast life history plus red noise may be

20 a potent factor producing sudden population disappearances. Using a simple mathematical

21 technique with the classic Rozenzweig-MacArthur consumer resource model, we created an

22 experiment varying the consumer's maximum population growth rate, effectively varying the

23 consumer's life history along the slow-fast continuum. Initial slowing of the consumer's growth

24 rate increased the stability of the consumer-resource model with added stochasticity. Slowing

25 the consumer's growth rate further and reddening the noise decreased the stability with

26 population disappearances occurring frequently. Taken together, we have shown that slow life

27 histories can both stabilize or destabilize interactions depending on the relative speeds within

28 the interaction. Disconcertingly with a reddening world, larger and slower organisms may be

29 especially prone to experiencing transient-driven sudden population disappearances.

\section{Keywords}

31 Slow-fast, life history, stability, transients, allometry, consumer-resource, red noise,

32 stochasticity, canards 


\section{Introduction}

34 The sudden or unexpected loss of a species has been observed both empirically and

35 theoretically. These dramatic population disappearances include the collapse of Atlantic cod

36 (Gadus morhua) fisheries in Newfoundland [1] and the abrupt decline of blue walleye (Sander vitreus glaucus) [2]. Although some population disappearances can be caused by a precipitous change in an environmental factor, such as overfishing in the case of the Atlantic cod fisheries [1], disconcertingly, even a small gradual change in a variable can cause a sudden population disappearance. The blue walleye decline, for example, is thought to have been caused by the slow onset of eutrophication [2] and is akin to deterministic theoretical arguments for population disappearances that are driven by slow extrinsic factors [3-5]. Although interesting, evidence for completely deterministic population disappearances may be hard to find in a variable or "noisy" world. Yet intriguingly, noise combining with deterministic species interactions could be a driving force behind population disappearances [6].

Researchers have started examining how noise - especially red noise (see Box 1 for definitions) - can exacerbate population disappearances. Specifically, climate change is reddening environmental noise by increasing the spatial and temporal autocorrelation of climate variables $[7,8]$ which can act to lengthen periods of suboptimal conditions for organisms [9]. Under extended suboptimal conditions, populations are driven to smaller and smaller numbers with demographic stochasticity often hastening local extinction [9]. The responsiveness of the organism including growth rate and the level of compensatory dynamics through density dependence can dictate the ability of the organism to escape extinction where highly responsive (high growth rate or over-compensatory dynamics) organisms are more likely to escape extinction when experiencing reddened noise [9-11]. Although red noise can lead to longer suboptimal conditions, red noise may also be expected to effectively enlarge the period and magnitude of a perturbation in any direction, potentially overwhelming the organism's density dependent compensatory responses that would normally bring the populations back to equilibria. The nonlinear dynamics away from the equilibria (hereafter referred to as non-local nonlinear dynamics, see Box 1) may be important in causing sudden population disappearances. Indeed, as illustrated by van der Bolt et al. [12] and Laakso et al. [13] who found that reddened noise can lead to critical transitions and reddened noise interacts with deterministic nonlinear dynamics to impact population extinction risk, reddened noise is useful to examine how noise and non-local nonlinear dynamics could cause sudden population disappearances.

67 Furthermore, researchers have been intrigued by how population life history traits may tie into and variability. Slow life histories are often associated with slow population growth rate (low r), large body size, and long generation times while fast life histories tend to exhibit fast population growth, small body size, and short generation times (Figure 1a) [14-17]. In general, theoretical research has found that faster organisms tend to produce overcompensatory dynamics and instability compared to slower organisms $[18,19]$. 
Consistent with this, empirical research has found negative correlations between population variability (one measure of stability) and both body size and generation time, and positive correlations between population variability and growth rates across multiple taxa and kingdoms [20-23]. Taken together, larger and slower organisms appear capable of buffering small perturbations better than small and fast organisms; however, especially when perturbations are reddened or auto-correlated, this same slow growth response may ensure that slow organisms are more likely to be pushed away from the equilibria.

These collective results suggest a gradient of stability along the slow-fast life history continuum (Figure 1b). Specifically, small fast-growing species are likely to show strong unstable over-compensatory responses. Slowing the life history speed by a small amount is likely stabilizing because the organisms will be deterministically stable $[18,19]$ and capable of buffering small perturbations near the equilibrium. However, slowing the life history speed by a large amount is likely destabilising because the organism's slow growth rates allow them to be easily moved away from the equilibrium, especially by autocorrelated or large perturbations $[24,25]$. Once a population is moved away from equilibrium they become subject to the vagaries of different nonlinear dynamics that can fundamentally alter the outcomes of the system including transient and sudden extinction. Although slowing the life history by a small amount may be initially stabilising, slowing the life history by a large amount plus reddening climate signals may be a hotbed for finding nonlinear transient dynamics that produce sudden population disappearances.

Here, we examine this potential stability gradient along the slow-fast life history continuum with a specific focus on sudden population disappearances in a reddened world. Specifically, we examine the consumer-resource interaction - a fundamental building block of whole communities - because it has been well described by allometric arguments that are conducive to slow-fast theory $[26,27]$. We use a longstanding and elegant technique called slow-fast modeling to scale the intrinsic maximum growth rate of the consumer so that we can vary life history along the slow-fast continuum (Figure 1a). This slow-fast modeling technique allows us to examine how changing the consumer's growth rate impacts the stability of the consumer-resource interaction while experimentally controlling the underlying deterministic dynamics (Figure 1b). We examined the stability implications in the consumer's growth rate through the classic linear stability analysis and through adding stochasticity ranging from white to red noise. Overall, we show that life history as predicted sets up a stability gradient with slow life histories especially sensitive to sudden population disappearances when perturbed by reddened noise. 
a)

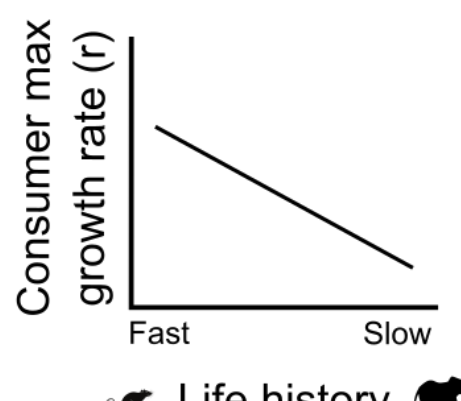

b)

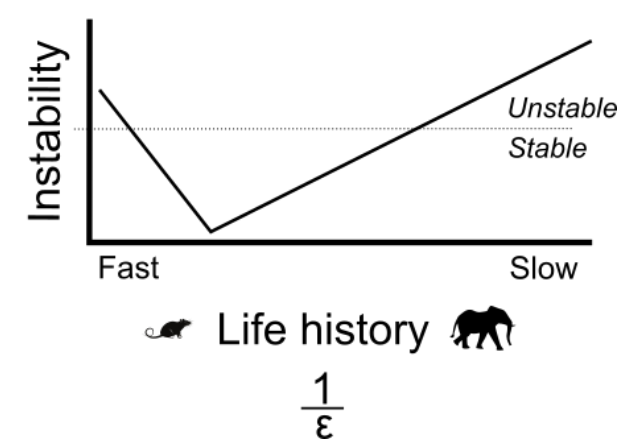

107 Figure 1 a) Comparison of empirical relationship between the slow-fast life history continuum 108 and consumer maximum growth rate, $r$ [14] with the equivalent relationship between $1 / \varepsilon$ (as a 109 stand in for life history) and consumer maximum growth rate, $r$, in the consumer-resource 110 model. In the consumer-resource model, $\varepsilon$ scales the $e \cdot a_{\max }$ and $m$ parameters in the 111 consumer equation such that consumer maximum growth rate $\left(e \cdot a_{\max }-m\right)$ decreases with 112 increasing $1 / \varepsilon$ where $e$ is the efficiency or conversion rate of consumed resources into new 113 consumers, $a_{\max }$ is the maximum rate of resources that can be consumed $(1 / h)$, and $m$ is the 114 mortality rate of the consumer. b) Conceptual illustration of hypothesized relationship between 115 life history speed and instability. Hypothesized relationship is similar to the check mark 116 relationship between energy flux and the maximum real eigenvalue in classic consumer117 resource theory. 


\section{Box 1 Key terms and definitions}

Stochasticity/Noise: In modeling, stochasticity/noise is considered to be all that is left out of a model (the deterministic skeleton which describes the process of interest) [6]. Modelers introduce stochasticity back into a model as a simplification of all the other processes happening at multiple scales. These other processes can either be intrinsic where variables of interest are averages of lower-level processes or extrinsic where stochasticity represents the effect of other variables and processes not explicitly modelled [6].

Red noise: Red noise is when noise from time points close together are similar (positively autocorrelated). Red noise is the opposite of blue noise where noise from time points close together are completely different (negatively autocorrelated). White noise is in between red and blue noise where noise is random with respect to time. When examining the power spectrum of the different noise types, red noise exhibits decreasing power with increasing frequency, white noise exhibits equal power across all frequencies, and blue noise exhibits increasing power with increasing frequency.

Stability: Stability can have many definitions [28]. Here, we define stability using the local linear perturbation analysis. We also expand the definition of stability to include stochasticity where unstable dynamics under stochasticity include oscillations that take dynamics close to extinction (similar to variability-driven species collapse [29]).

Non-local nonlinear dynamics: Nonlinear dynamics occur in systems that react disproportionately to initial conditions or a small perturbation. These dynamics can include chaos and limit cycles. In this study, we differentiate between local and non-local nonlinear dynamics. Local nonlinear dynamics are dynamics when system trajectories are close to the equilibria in phase space. Non-local nonlinear dynamics are dynamics when system trajectories are far from equilibria. We differentiate between local and non-local nonlinear dynamics because the dynamics will usually be different.

Transient dynamics: Transient dynamics occur before the asymptotic end points (in other words when the attractors have been reached). Specifically, transient dynamics are a persistent dynamical pattern that lasts for several generations but that is not the stable longterm end point dynamics [30]. In ecological research, transients are important because human observation and conservation management of ecosystems occur over short time scales relative to the time scales required to reach the asymptotic end points [30,31]. One potential outcome from transient dynamics are sudden population disappearances (see Figure 2) that would have been missed from using solely an asymptotic dynamics analysis.

Quasi-cycle: Quasi-cycles are a result of stochasticity resonating with damped oscillations surrounding an interior equilibrium $[6,32,33]$. Frequencies in the stochastic noise that most closely resemble the period of the damped oscillations are amplified. Thus, a power spectrum would show all frequencies with the frequency of the damped oscillations having the highest power [32]. In contrast, a power spectrum of deterministic damped oscillations would show a 
single frequency [6]

Quasi-canard: A quasi-canard is a stochastically induced version of a deterministic canard. A deterministic canard is when a system's solution follows an attracting manifold, passes over a critical point along this manifold, and then follows a repelling manifold [34]. In the consumerresource model, the canard solution slowly follows the resource isocline (the attracting manifold) until the maximum point of the isocline is reached (the critical point), then the solution quickly jumps to the consumer axis before slowly following the consumer axis (the repelling manifold). Finally, the solution quickly jumps back to the resource isocline and repeats the canard cycle. When stochasticity is introduced, trajectories can combine both small oscillations around the equilibria and large relaxation oscillations qualitatively similar to deterministic canards. Generally, these patterns are called mixed mode oscillations [34]. However, we use the term quasi-canards in this study as a comparison to quasi-cycles.

118 Methods

119 Model

120 In this study, we used the classic Rosenzweig-MacArthur consumer-resource model but with

121 the addition of the parameter $\varepsilon$ to separate a slow and a fast variable. In this case, the

122 resource is the "fast" variable and the consumer is the "slow" variable.

123

$\frac{d R}{d t}=r R\left(1-\frac{R}{k}\right)-\frac{a R C}{1+a h R}$

124

$\frac{d C}{d t}=\varepsilon\left(\frac{e a R C}{1+a h R}-m C\right)$

125

126

127

128

129

130

131

132

133

134

135

136

137 where $r$ is the intrinsic growth rate of the resource (R), $k$ is the carrying capacity of the resource, $a$ is the attack rate of the consumer (C), $e$ is the efficiency or conversion rate of consumed resources into new consumers, $h$ is the handling time, and $m$ is the consumer mortality. For all analyses, $r=2.0, k=3.0, a=1.1, h=0.8$, and $m=0.4$.

Note, $\varepsilon$ scales the consumer maximum growth rate $\left(e \cdot a_{\max }-m\right.$ where $a_{\max }$ is $\left.1 / h\right)$ because as resources increases to infinity the type II functional response $\left(R /\left(R_{0}+R\right)\right.$ where $R_{0}$ is $\left.1 / a h\right)$ asymptotes at 1 leaving $e \cdot a_{\max }-\mathrm{m}$. Therefore, $a_{\max }$ is the maximum rate of resources that can be consumed. As a further illustration of this point, the $r$ in the classic logistic equation is equivalent to the consumer-resource maximum growth rate, $e \cdot a_{\max }-m$, because when resource density is small there is no density dependence.

Overall, we use the parameter, $\varepsilon$, to scale the consumer's intrinsic growth rate and effectively manipulate the life history of the consumer along a slow-fast continuum (relative to the resource) (Figure 1a). 


\section{Small slowing of consumer growth rate}

Researchers often use the term excitability to describe the organism's overcompensation response leading to damped oscillations. The excitability of the consumer-resource interaction has been mathematically studied within the context of changing energy fluxes into the consumer $[21,29]$. Non-excitable dynamics are when the eigenvalues are real and the model exhibits monotonic dynamics. In contrast, excitable dynamics are when the eigenvalues are complex and the model exhibits oscillatory dynamics. The divide between excitable and nonexcitable is the real to complex divide in the eigenvalues of the model from the interior equilibrium. Thus, we can examine how slowing the consumer's growth rate (increasing $1 / \varepsilon$ ) by a small amount impacts the real-complex divide. We numerically calculated the real to complex divide for each value of $1 / \varepsilon$ between 0.01 and 10 - with a step size of 0.001 in $\varepsilon$ (package LinearAlgebra.jl). For each $1 / \varepsilon$, we found the efficiency value, $e_{R / c}$, where the real/complex divide occurs (i.e. after $1 / \varepsilon$ is set, the efficiency value is increased from the efficiency value that produces the transcritical bifurcation until the eigenvalues switch from real to complex, akin to finding the value at which the tip of the checkmark occurs in Gellner and McCann [19]). This efficiency value was then used to calculate the proportion of efficiency "parameter space" that produces real eigenvalues (Proportion Real) by subtracting the $\mathrm{real} / \mathrm{complex}$ divide efficiency value $\left(e_{R / C}\right)$ from the efficiency value at the Hopf bifurcation $\left(e_{\text {Hopf }}\right)$ and then dividing this value by the efficiency parameter distance between the deterministic Hopf $\left(e_{\text {Hopf }}\right)$ and transcritical $\left(e_{\text {transcritical }}\right)$ bifurcation efficiency values (note $\varepsilon$ does not change where the Hopf and transcritical bifurcation occur):

Proportion Real $=\frac{e_{\text {Hopf }}-e_{R / C}}{e_{\text {Hopf }}-e_{\text {transcritical }}}$

Existing theory has found that stochastic perturbations can resonate with the excitability of the consumer-resource model to extend the range of cyclic dynamics [29,32] producing what has been termed quasi-cycles. These quasi-cycles generally do not threaten persistence because they are small relative to the deterministic limit cycles. Nevertheless, if slowing the organism's life history reduces the excitability of the consumer-resource interactions, we should find that a slowed consumer-resource interaction does not readily exhibit quasi-cycles, thus increasing stability. To examine how slowing the consumer impacted quasi-cycles, we ran 1000 simulations each of the consumer-resource model with $1 / \varepsilon$ values of 1 and 1.667 and where the consumer variable was perturbed every time step with normally distributed noise (with mean 0.0 and variance 0.001) (package DifferentialEquations.jl v6.20.0, Algorithms: Vern7 \& Rodas 4 with automatic stiffness detection). We calculated the autocorrelation of the last 1000 time steps of each simulation for lags between 0 and 40 (function autocor, package StatsBase v0.33.13). We then calculated the average autocorrelation value for each lag across the 1000 simulations for each simulation set with a different $1 / \varepsilon$ value. As suggested by Pineda-Krch et al. [32], we used autocorrelation to measure the manifestation of quasi-cycles where an autocorrelation function from quasi-cycles would show pronounced low-amplitude oscillations that decrease in amplitude with increasing lags. 


\section{Large slowing of consumer growth rate}

To examine how slowing the consumer's growth rate by a large amount affects the stability of the consumer-resource interaction, we first extended the real/complex divide analysis above to larger values of $1 / \varepsilon$. Next we examined the prevalence of extinction prone dynamics in stochastically perturbed simulations of the consumer-resource model with varying $\varepsilon$ and efficiency values. These extinction prone dynamics are either a quasi-canard or settling on the axial solution (the resource carrying capacity) (Figure 2). A quasi-canard creates a similar pattern to the canard pattern that is found when the consumer isocline sits to the left of the Hopf bifurcation point in a deterministic consumer-resource model [35]. For each simulation, the consumer was perturbed with normally distributed noise (with mean 0.0 and variance 0.001 ) every time step (package DifferentialEquations.jl v6.20.0, Algorithms: Vern7 \& Rodas4 with automatic stiffness detection). For each combination of $\varepsilon$ and efficiency value, we calculated the proportion of simulations that exhibited at least one quasi-canard. To calculate the proportion of simulations with quasi-canards, we created an algorithm to check whether a time series contained a quasi-canard (or landed on the axial solution). More details of this algorithm can be found in the Supporting Information, but in short the algorithm includes a return map at the maximum point of the resource isocline where canards and quasi-canards must pass through. The algorithm also includes boxes along the attracting and repelling manifolds (the right side of the resource isocline and the consumer axis respectively) through which a quasi-canard should pass. Note, this first analysis considered only quasi-canards because we wanted to establish the potential for quasi-canards with varying $\varepsilon$ and efficiency values. $1 / \varepsilon$ was varied from 6.667 to 1,000 . Our lower bound for $1 / \varepsilon$ was set at 6.667 because no quasi-canards can be found below this point. We set efficiency as either 0.5 (where the deterministic dynamics are non-excitable), 0.65 (where the deterministic dynamics are excitable but still locally stable), and 0.7 (where the deterministic dynamics are excitable, but still locally stable, and close to the Hopf bifurcation). We ran 1,000 simulations with two maximum time steps $(6,000$ and 24,000$)$ for each combination of $\varepsilon$ and efficiency. Two maximum time steps were compared to show that increasing the maximum time steps increases the proportion of quasi-canards that can be found due to the probabilistic nature of the stochastic dynamics. 


\section{Population disappearance}
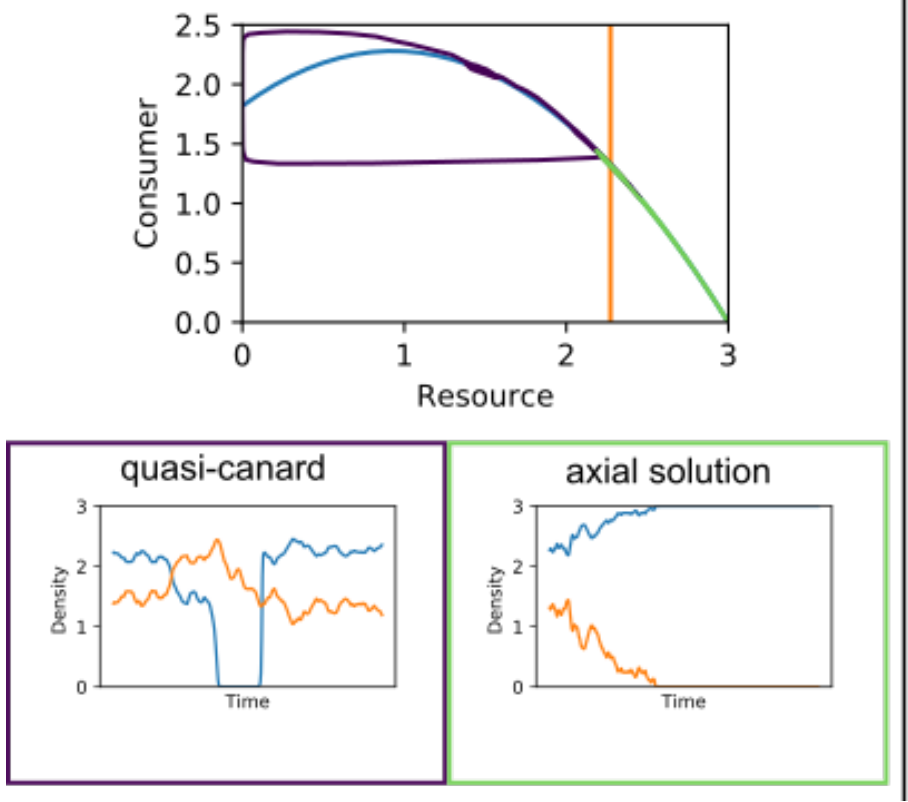

Stable
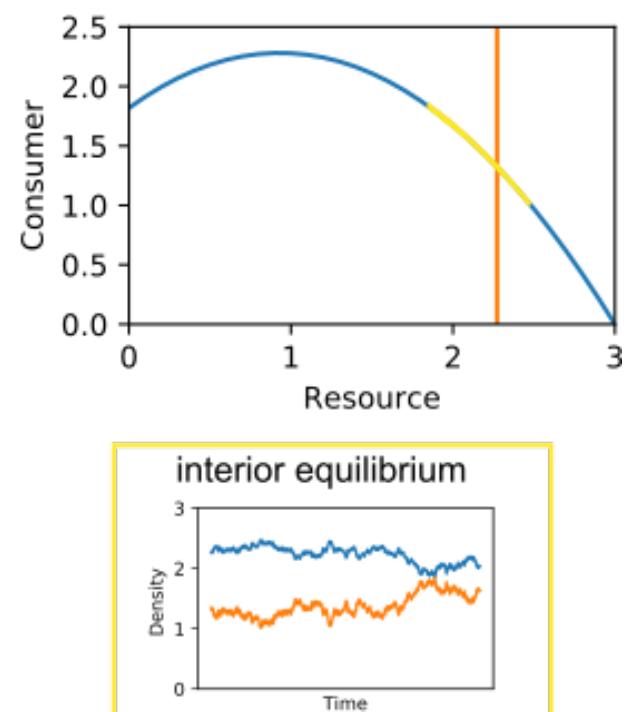

207 Figure 2 Possible qualitative dynamics when $1 / \varepsilon$ is large (small consumer growth rate) and the consumer variable is stochastically perturbed. Blue is for the resource and orange is for the consumer. The dynamics are split into population disappearance and stable. The two possible population disappearance dynamics are quasi-canard and reaching the axial solution (the resource carrying capacity). For the quasi-canard, note the trajectory moving up the resource isocline before jumping to the consumer axis after passing the maximum of the resource isocline. The trajectory then slowly moves down the consumer axis and finally jumps to the resource isocline. The stable dynamic is noise around the interior equilibrium.

\section{To examine how reddened noise impacts the consumer-resource interaction when the} consumer growth rate has been slowed by a large amount, we changed the stochastic noise from white noise to red noise for different combinations of $\varepsilon$ and efficiency. We examined the effect of reddened noise with only large decreases in consumer growth rate because small decreases in consumer growth rate do not produce quasi-canards. For each combination of noise type, $\varepsilon$, and efficiency, we ran 1000 simulations and calculated the proportion of simulations that exhibited at least one quasi-canard, the proportion of simulations that reached the axial solution but did not exhibit quasi-canards, and the proportion of simulations that did not exhibit quasi-canards or reached the axial solution (package DifferentialEquations.jl v6.20.0, Algorithms: Vern7 \& Rodas4 with automatic stiffness detection). We used an $A R_{1}$ process to create red noise and scaled the variance of the red noise to the variance from the original white noise using the technique in Wichmann et al. [36] where the ratio of white noise to red noise variances is used to scale individual noise values in the red noise sequence.

All analyses were done using julia version 1.7.0. [37] 


\section{Results}

\section{Small slowing of consumer growth rate}

232 Small increases in $1 / \varepsilon$ moves the real-complex divide towards the Hopf bifurcation and thus,

233 increases the proportion of efficiency "parameter space" that produces real eigenvalues

234 (Figure 3a). In other words, increasing $1 / \varepsilon$, reduces the excitability of the system. We also

235

236

237 proved using the non-dimensional type I version of the Rosenzweig-MacArthur consumerresource model that increasing $1 / \varepsilon$, increases the efficiency value where the real/complex divide occurs (see SI Section Proof of excitability decreasing when slowing the consumer).

With a small increase in $1 / \varepsilon$ (from 1 to 1.667), the average autocorrelation function (ACF) line flattens out indicating reduced manifestation of quasi-cycles (Figure $3 \mathrm{~b} \& \mathrm{c}$ )

a)

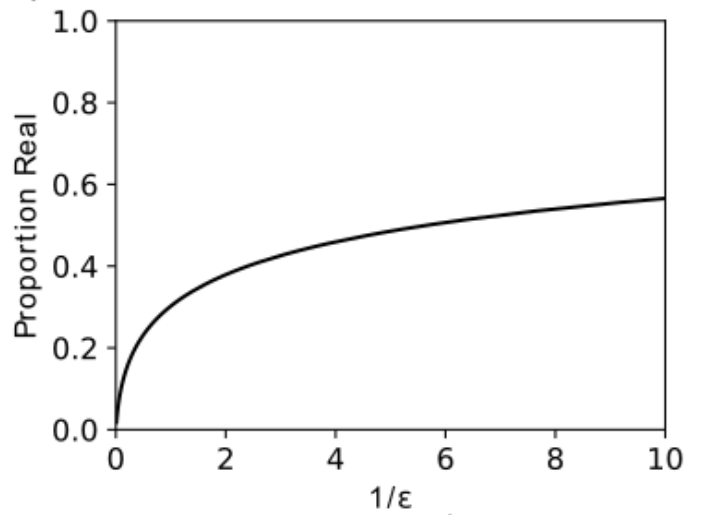

b)

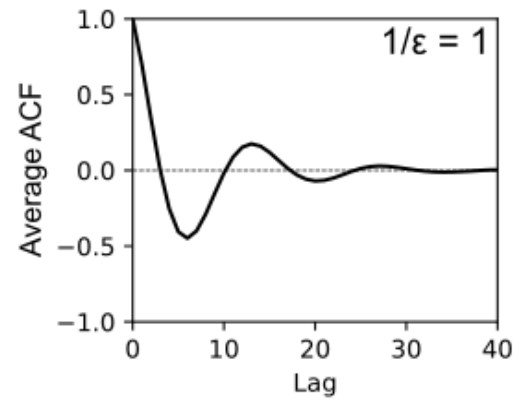

c)

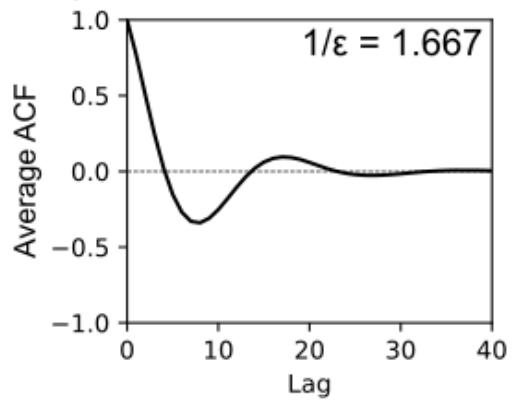

240 Figure 3 Slowing the consumer by a small amount stabilizes the consumer-resource $1 / \varepsilon$ value where the full efficiency "parameter space" corresponds to the distance between the efficiency values that produce the transcritical and Hopf bifurcations. b) \& c) average ACF for each lag value for 1000 simulations of the consumer-resource model perturbed each time step by normally distributed noise (with mean 0.0 and variance 0.001 ) with $1 / \varepsilon$ values of 1 and 1.667 respectively. 


\section{Large slowing of consumer growth rate}

Large increases in $1 / \varepsilon$ continued to move the real-complex divide towards the Hopf bifurcation with the proportion of efficiency "parameter space" that produces real eigenvalues asymptoting just under 1.0 (Figure 4a).

As long as $1 / \varepsilon$ was large enough, we found quasi-canards when the consumer-resource model had small efficiency values producing non-excitable and locally stable dynamics (small efficiency values) (Figure 4b). When the consumer-resource model had larger efficiency values model producing excitable but still locally stable dynamics, small and large values of $1 / \varepsilon$ produced quasi-canards (Figure $4 c \& d$ ). For very large $1 / \varepsilon$ values, the proportion of simulations that exhibited quasi-canards decreased (Figure $4 c \& d$ ). However, this was because the consumer growth rate was so slow that the time required to find a quasi-canard increased as evidenced by comparing the two dashed lines for 6,000 and 24,000 time steps.

Generally, population disappearances (quasi-canard or axial solution) were more likely to be found when the consumer was perturbed by reddened noise compared to when the consumer was perturbed by white noise (Figure 5). For a small value of $1 / \varepsilon$, a non-excitable, locally stable consumer-resource model did not exhibit any quasi-canards regardless of the noise autocorrelation but did reach the axial solution for reddened noise (Figure 5a). When the consumer-resource model had a small $1 / \varepsilon$ value and was excitable but still locally stable (larger efficiency values), increasing the noise autocorrelation increased the proportion of simulations that exhibited quasi-canards (Figure 5 b \& c). For a large value of $1 / \mathcal{E}$, a nonexcitable, locally stable consumer-resource model exhibited quasi-canards and axial solutions when the noise autocorrelation was high (Figure $5 \mathrm{~d}$ ). When the consumer-resource model had a large $1 / \varepsilon$ value and was excitable but still locally stable (larger efficiency values), quasicanards were mostly found but higher noise autocorrelation increased the proportion of simulations where the axial solution was reached (Figure 5 e \& f). 


\section{a)}

b)

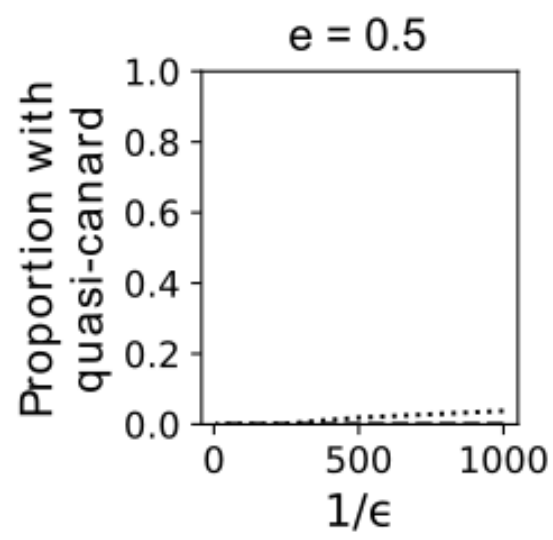

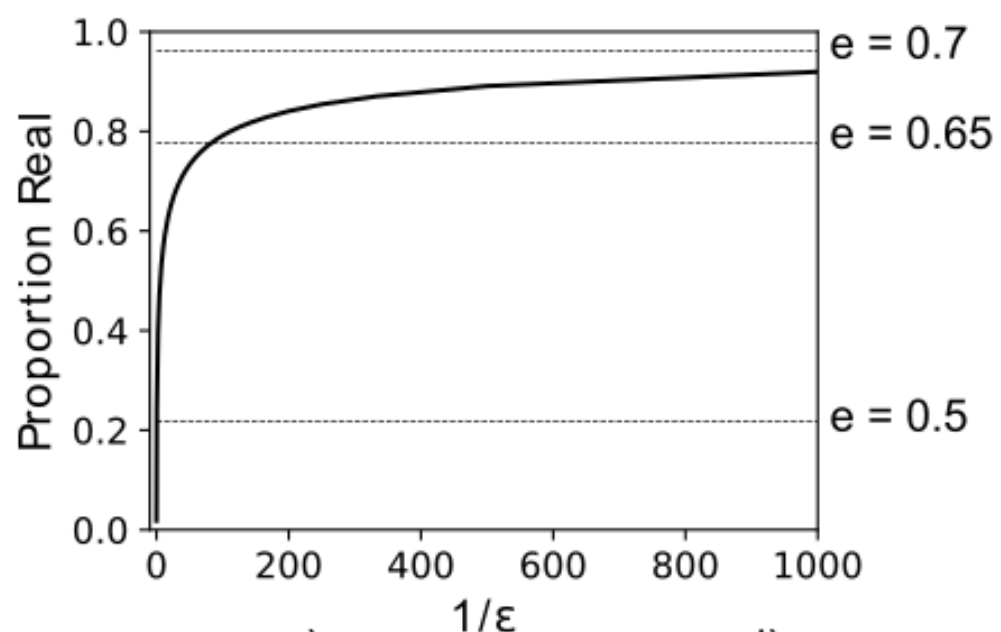

c)

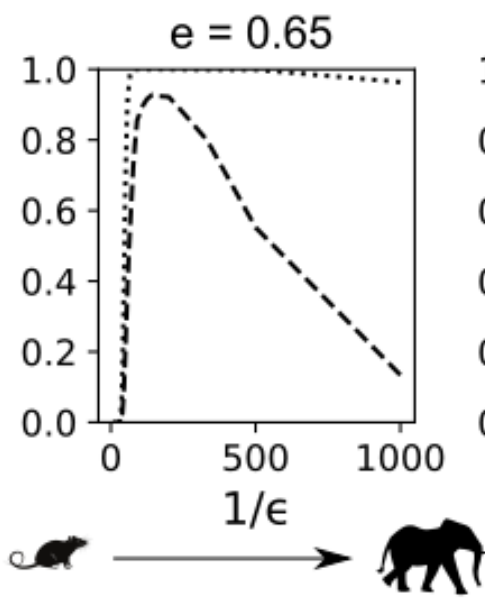

d)

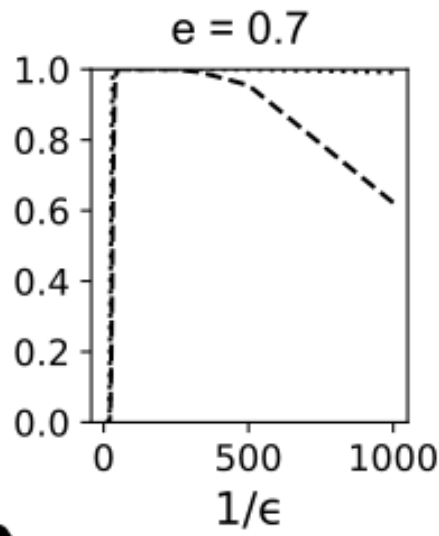

273 Figure 4 Quasi-canards can be found for a variety of efficiency values within certain 274 boundaries of $\varepsilon$ parameter space. a) Proportion of efficiency "parameter space" that produces completely real eigenvalues for each $1 / \varepsilon$ value. Thin dashed lines depict the effective proportion of efficiency "parameter space" for each efficiency value that produces b), c) \& d) (e.g. $\left.\frac{e_{\text {Hopf }}-0.5}{e_{\text {Hopf }}-e_{\text {transcritical }}}\right)$. With an efficiency value of 0.5 , the model is essentially always real regardless of the $1 / \varepsilon$ value. With an efficiency value of 0.65 , the model is complex with smaller values of $1 / \varepsilon$ and real with larger values of $1 / \varepsilon$. With an efficiency value of 0.7 , the model is always complex regardless of the $1 / \varepsilon$ value. b), c), d) proportion of 1000 simulations per value of $1 / \varepsilon$ that exhibited quasi-canards with constant efficiency values of $0.5,0.65,0.7$ respectively. Bold and thin dashed lines correspond to 6,000 and 24,000 time steps respectively. 


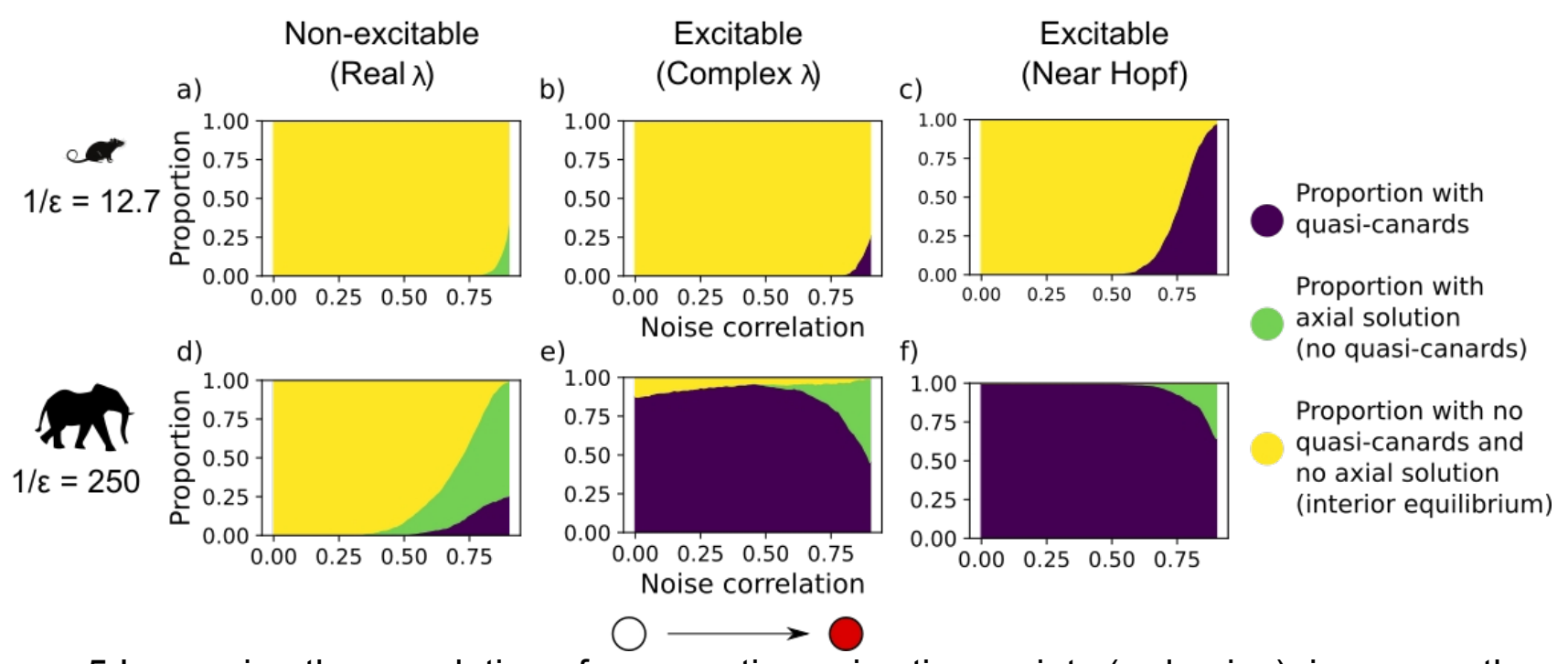

Figure 5 Increasing the correlation of consecutive noise time points (red noise), increases the proportion of simulations exhibiting population disappearances where quasi-canards (purple) and axial solution (green) would be population disappearances. For a), b), c), d), e), \& f) the noise $\left(A R_{1}\right.$ process) correlation was varied from 0.0 to 0.9 . The top row $\left.\left.(a), b\right), \& c\right)$ ) had a $1 / \varepsilon$ value of 12.7 . and the bottom row (d), e), \& f) ) had a $1 / \varepsilon$ value of 250 . Each column of plots had efficiency values of $0.5,0.65$, and 0.7 respectively.

\section{0}

\section{Discussion}

Using the simple technique of changing the time scales within the Rosenzweig-MacArthur consumer-resource model, we set up a biologically motivated mathematical experiment exploring the stability of faster and slower life histories with an emphasis on whether slow life history strategies can be destabilized into sudden population disappearances when pushed by large perturbations such as red noise. This experiment was achieved because changing the time scales of the consumer using $\varepsilon$ also changes the maximum consumer intrinsic growth rate, a population parameter that in nature is negatively correlated with the fast to slow life history continuum [14]. With a small decrease in consumer growth rate, stability increases. With a larger decrease in consumer growth rate, stability decreases because the consumer and resource are more susceptible to sudden population disappearances. Indeed, for a broad range of epsilon values $(0.001 \sim 0.15)$ that are indicative of slower life history strategies (low mass specific metabolic rate, weak growth rates or weak numerical responses), we have found that sudden population disappearances can occur even when the consumer interacts weakly with the resource and exhibits monotonic dynamics (i.e. real eigenvalue and consumer isocline lies well onto the right half of the resource isocline [29]). Furthermore, increasing the correlation of noise increased the likelihood of sudden population disappearances. Although the range in epsilon is different, Yodzis \& Innes's [26] biologically plausible consumer-resource model similarly exhibits quasi-canards (see SI Section "Biologically plausible parameters" \& SI Figure 3). Consequently, our results are general and suggest that such transient-driven instability ought to occur under increasingly reddened perturbations with climate change. 
Our results do confirm a gradient in stability along the slow-fast life history continuum. With a small decrease in consumer growth rate, the excitability of the consumer-resource interaction decreases with concomitant reduced manifestation of quasi-cycles in the face of tiny perturbations every time step. However, those same tiny perturbations cumulatively can produce large instability and population disappearances when the consumer has a small growth rate. Another way of conceptualizing this stability gradient is a trade off along the slowfast continuum between low short-term stability but high resistance to large perturbations for fast organisms and high short-term stability but low resistance to large perturbations for slow organisms. This trade-off is corroborated by other researchers where organisms with slow growth rates and long generation times are thought to buffer small disturbances well but can be unstable depending on the type and strength of perturbations [24,25]. One caveat is that increasing the energy flux of the system appeared to reduce the stability gradient along the slow-fast continuum as shown by increasing proportions of quasi-canards for locally excitable consumer-resource models. A common finding is that increasing the excitability of an interaction - usually through increasing the energy flux of an interaction (i.e. ae/m) - leads to higher instability and variability $[21,38]$. Thus, adding noise to an already highly excitable interaction can lead to transient-driven sudden population disappearances. Overall, there appears to be a gradient in stability along the slow-fast continuum that is mediated by energy flux in the system.

The combination of life history and red noise significantly increased the opportunities for transient dynamics and sudden population disappearances. Reddening the noise in our consumer-resource model increased the likelihood of sudden population disappearances for both fast and slow organisms. However, the onset of sudden population disappearances occurred with less autocorrelation for slower organisms. To understand this pattern, we must examine the relative time scales of the autocorrelation and the system population processes [6]. Slowing the life history is effectively increasing the time scale of the population response processes, and thus the system will stay for longer in the phase space region the system was pushed into after a perturbation. Increasing the autocorrelation increases the time scale of the perturbations, and thus the perturbation effectively feels larger to the system. Another way to understand how slow life histories and reddened noise interact, is to use the analogy of a rusty ratchet. White noise is akin to a ratchet without a pawl and can spin in any direction but red noise is akin to a ratchet with a pawl that has a tendency to spin in one direction for a period of time (Figure 6). Because the reddened noise has a tendency to produce similar values for a period of time, reddened noise consistently pushes the dynamics of the consumer-resource model far from the local area around the stable equilibria. The slow life history is akin to rust in the ratchet which slows the spinning speed and in the system increases the difficulty for the trajectories to return to the local area around the equilibria. Related research has examined how multiple discrete disturbances can kick dynamics out of basins of attraction to produce different dynamical outcomes (flow-kick dynamics: Meyer et al. [39]). These researchers found that rare but large disturbances can have the same effect as frequent and small disturbances. Reddened noise is similar to frequent and small disturbances, pushing dynamics far from their attracting equilibria (the kicks in flow-kick dynamics). Furthermore, slowing life histories reduces the relative time available for organisms to respond to the disturbances (the flow in flow-kick dynamics), thus adding to pushing dynamics far from their attracting equilibria. As illustrated by our model and by 
comparisons to flow-kick dynamics, reddened noise plus slow life histories have huge potential for low stability and sudden population disappearances.
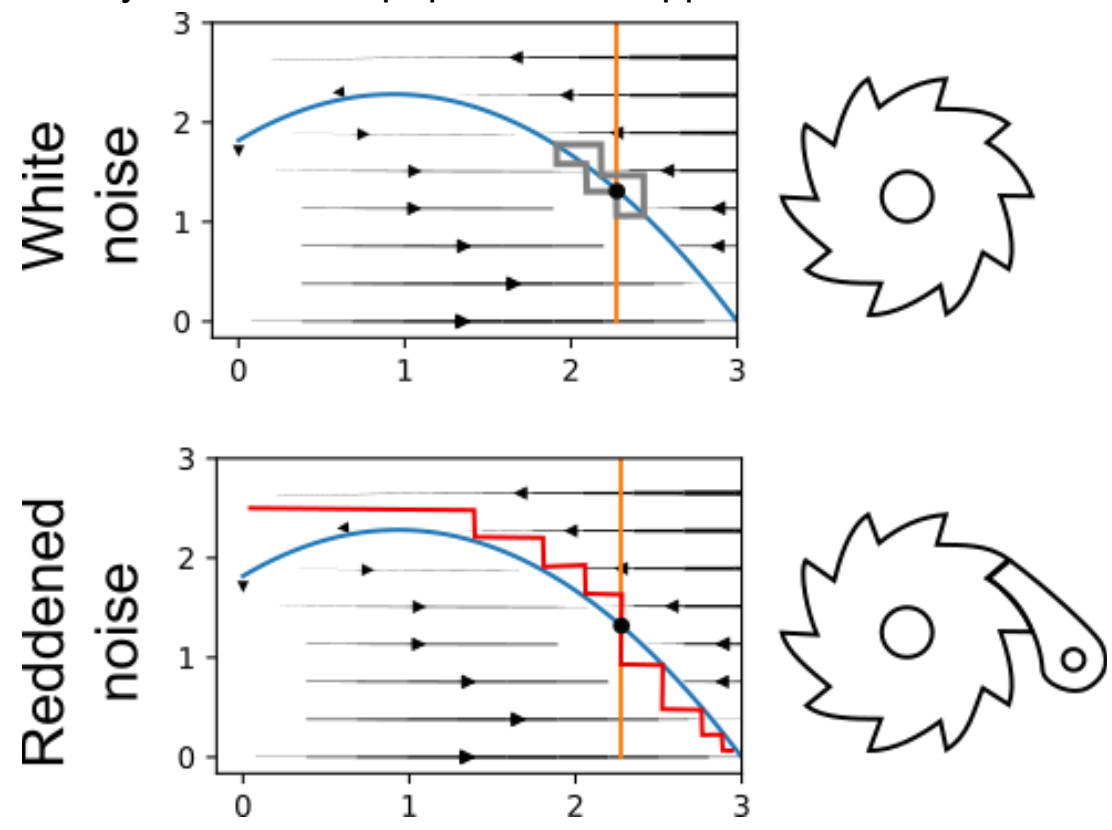

359

360

361

362

363

364

365

366

367

368

369

370

371

372

373

374

375

376

377

378

379

380

381

382

383

Figure 6 Illustration of simplified trajectories with white noise and red noise imposed on consumer and resource isoclines and vector field diagram when $1 / \varepsilon$ is large. We use the analogy of a rusty ratchet to illustrate the interaction of slow life histories with reddened noise. White noise is similar to a ratchet wheel without the pawl (can spin in any direction) and reddened noise is similar to a ratchet with the pawl (has the tendency to spin in one direction). Slow life history is akin to rust in the ratchet which slows the spinning speed.

Intriguingly, our examination of slow life histories with stochasticity exhibiting sudden population disappearances is a further example of how stochasticity is immensely useful in ecological research to understand the full nonlinear dynamics of ecosystems $[6,33,40]$. First, stochasticity can act to uncover underlying processes (Boettiger [6] coined the phrase noise the informer for this phenomenon). Similar to quasi-cycles where stochastic resonance is visible in advance of a Hopf bifurcation, the quasi-canards also occur in advance of the Hopf bifurcation after which deterministic canards occur. Although noise has the same effect of uncovering an imminent Hopf bifurcation leading to either deterministic cycles or canards, the mechanism producing the quasi-cycles and quasi-canards are different. The quasi-cycles are stochasticity interacting with local nonlinear dynamics (excitability). The quasi-canards are slow life histories plus reddened noise pushing dynamics towards non-local nonlinear dynamics. Second, stochasticity can be used to reveal differences in stability from what our normal linear stability analysis would find. One method to reveal these differences in stability is through using the stochastic equivalent of potential functions called quasipotentials where potential functions and quasipotentials can be simplified conceptually to the ball and cup analogy [41]. The quasipotentials for our model with different $1 / \varepsilon$ values show the stretching of the quasipotential along the resource isocline due to stochasticity, then the quasi-canard shape when efficiency is large enough, and finally the flattening of the quasipotential quasicanard shape with a larger $1 / \varepsilon$ value (see SI Figure 4). Linear stability analysis would solely 
384

385

386

387

388

389

390

391

392

393

394

395

396

397

398

399

400

401

402

403

404

405

406

407

408

409

410

411

412

413

414

415

416

417 Acknowledgments

418 We wish to thank members of the McCann laboratory for thoughts and feedback.

focus on the tiny region around the intersect of the consumer and resource isoclines. In contrast, the flattened quasipotentials reveal other possible dynamics (quasi-canards) in addition to the stable interior equilibria. Overall, stochasticity can both produce and reveal divergent dynamical outcomes from the normal linear stability analysis.

Sudden population disappearances from transient dynamics may not necessarily lead to the full extirpation of a species. Instead, the interaction may be lost with detrimental cascading consequences on the ecosystem [42]. In our study, we used a consumer-resource model that represents a fundamental interaction within ecosystems [43]. Our model shows that even with moderate differences between relative speeds of the consumer and resource, the conditions necessary to lose the interaction - small abundances of both organisms - can occur. For the quasi-canard, the initial small abundances of the resource can lead to continued small abundance of consumers. Alternatively, when the dynamics reach the axial solution, the consumer is completely lost with the resource remaining. Either way, the consumer-resource interaction is lost. Overall, interactions with large differences in relative life history speeds or interactions that experience red noise are likely prone to sudden population disappearances and at the very least to interaction loss due to the large nonlinear fluctuations.

\section{Conclusion}

Through the technique of varying life history growth rates along the slow-fast continuum within the classic Rosenzweig-MacArthur model, we have shown that life history has interesting stability consequences depending on the speed. Slowing the life history can increase stability in the face of many tiny perturbations up to a point, after which slowing the life history can dramatically decrease stability with the potential for sudden population disappearances. Adding positively correlated noise to an already slowed consumer increases the occurrence of sudden population disappearances because the system is easily and frequently pushed away from the stable equilibria into transient dynamics. Although the consumer-resource motif is fundamental and can inform much of food web theory, our study examines a single consumer-resource interaction. The interaction of many more organisms with varying life histories is required. Furthermore, other examples of transient-driven sudden population disappearances should be explored especially in the context of slow life histories and reddened noise. Taken together, we have shown how life history along the slow-fast life history continuum can impact the stability of systems and shown how human-caused red noise will disproportionately impact slow living organisms through transient-driven sudden population disappearances. 


\section{Funding}

420 CJGG was supported by a Natural Sciences and Engineering Research Council of Canada

421 (NSERC) CGS-D. Financial support was provided by an NSERC Discovery Grant (400353)

422 and a CFREF Food from Thought grant (499075) to K. S. McCann.

\section{Author Contributions}

424 All authors contributed to the idea generation and analysis of the model. CJGG wrote the first

425 draft and all authors contributed to editing the manuscript.

\section{Code accessibility}

427 All code to reproduce the above analyses and figures are publicly available on GitHub and 428 have been archived on Zenodo (version 1.0).

\section{References}

1. Myers RA, Cadigan NG. 1995 Was an increase in natural mortality responsible for the collapse off northern cod? Can. J. Fish. Aquat. Sci. 52, 1274-1285. (doi:10.1139/f95-124)

2. Beeton AM. 1969 Changes in the Environment and Biota of the Great Lakes. In Eutrophication: Causes, Consequences, Correctives, pp. 150-187. Washington, DC: The National Academies Press. (doi:10.17226/20256)

3. McCann K, Yodzis P. 1994 Nonlinear dynamics and population disappearances. Am. Nat. 144, 873-879. (doi:10.1086/285714)

4. Schreiber SJ. 2001 Chaos and population disappearances in simple ecological models. J. Math. Biol. 42, 239-260. (doi:10.1007/s002850000070)

5. Hastings A, Wysham DB. 2010 Regime shifts in ecological systems can occur with no warning. Ecol. Lett. 13, 464-472. (doi:10.1111/j.1461-0248.2010.01439.x)

6. Boettiger C. 2018 From noise to knowledge: how randomness generates novel phenomena and reveals information. Ecol. Lett. 21, 1255-1267. (doi:10.1111/ele.13085)

7. Lenton TM, Dakos V, Bathiany S, Scheffer M. 2017 Observed trends in the magnitude and persistence of monthly temperature variability. Sci. Rep. 7, 5940. (doi:10.1038/s41598017-06382-x)

8. Di Cecco GJ, Gouhier TC. 2018 Increased spatial and temporal autocorrelation of temperature under climate change. Sci. Rep. 8, 14850. (doi:10.1038/s41598-018-33217$0)$ 
9. Schwager M, Johst K, Jeltsch F. 2006 Does red noise increase or decrease extinction risk? Single extreme events versus series of unfavorable conditions. Am. Nat. 167, 879888. (doi:10.1086/503609)

10. Ruokolainen L, Lindén A, Kaitala V, Fowler MS. 2009 Ecological and evolutionary dynamics under coloured environmental variation. Trends in Ecology \& Evolution 24, 555563. (doi:10.1016/j.tree.2009.04.009)

11. Lögdberg F, Wennergren U. 2012 Spectral color, synchrony, and extinction risk. Theor. Ecol. 5, 545-554. (doi:10.1007/s12080-011-0145-x)

12. van der Bolt B, van Nes EH, Bathiany S, Vollebregt ME, Scheffer M. 2018 Climate reddening increases the chance of critical transitions. Nature Clim. Change. 8, 478-484. (doi:10.1038/s41558-018-0160-7)

13. Laakso J, Kaitala V, Ranta E. 2004 Non-linear biological responses to environmental noise affect population extinction risk. Oikos 104, 142-148. (doi:10.1111/j.00301299.2004.12197.x)

14. Savage VM, Gillooly JF, Brown JH, West GB, Charnov EL. 2004 Effects of body size and temperature on population growth. Am. Nat. 163, 429-441. (doi:10.1086/381872)

15. Gaillard J -M., Yoccoz NG, Lebreton J -D., Bonenfant C, Devillard S, Loison A, Pontier D, Allaine D. 2005 Generation time: A reliable metric to measure life-history variation among mammalian populations. Am. Nat. 166, 119-123. (doi:10.1086/430330)

16. Réale D, Garant D, Humphries MM, Bergeron P, Careau V, Montiglio P-O. 2010 Personality and the emergence of the pace-of-life syndrome concept at the population level. Phil. Trans. R. Soc. B 365, 4051-4063. (doi:10.1098/rstb.2010.0208)

17. Healy K, Ezard THG, Jones OR, Salguero-Gómez R, Buckley YM. 2019 Animal life history is shaped by the pace of life and the distribution of age-specific mortality and reproduction. Nat. Ecol. Evol. 3, 1217-1224. (doi:10.1038/s41559-019-0938-7)

18. Stone L. 1993 Period-doubling reversals and chaos in simple ecological models. Nature 365, 617-620. (doi:10.1038/365617a0)

19. Gellner G, McCann KS. 2016 Consistent role of weak and strong interactions in high- and low-diversity trophic food webs. Nat. Commun. 7, 11180. (doi:10.1038/ncomms11180)

20. Gaston KJ, Lawton JH. 1988 Patterns in body size, population dynamics, and regional distribution of bracken herbivores. Am. Nat. 132, 662-680. (doi:10.1086/284881)

21. Rip JMK, McCann KS. 2011 Cross-ecosystem differences in stability and the principle of energy flux: Cross-ecosystem differences in stability. Ecol. Lett. 14, 733-740.

(doi:10.1111/j.1461-0248.2011.01636.x) 
22. Májeková M, de Bello F, Doležal J, Lepš J. 2014 Plant functional traits as determinants of population stability. Ecology 95, 2369-2374. (doi:10.1890/13-1880.1)

23. Röpke C, Pires THS, Zuanon J, Freitas CEC, Hernandes MC, Souza F, Amadio S. 2021 Growth-reproduction trade-off and fecundity regulate population stability in Amazon floodplain fishes. Freshwater Biol. , fwb.13702. (doi:10.1111/fwb.13702)

24. Sæther B-E et al. 2013 How life history influences population dynamics in fluctuating environments. Am. Nat. 182, 743-759. (doi:10.1086/673497)

25. Gamelon M, Gimenez O, Baubet E, Coulson T, Tuljapurkar S, Gaillard J-M. 2014 Influence of life-history tactics on transient dynamics: A comparative analysis across mammalian populations. Am. Nat. 184, 673-683. (doi:10.1086/677929)

26. Yodzis P, Innes S. 1992 Body size and consumer-resource dynamics. Am. Nat. 139, 1151-1175. (doi:10.2307/2462335)

27. Brose U, Williams RJ, Martinez ND. 2006 Allometric scaling enhances stability in complex food webs. Ecol. Lett. 9, 1228-1236. (doi:10.1111/j.1461-0248.2006.00978.x)

28. Donohue I et al. 2013 On the dimensionality of ecological stability. Ecol. Lett. 16, 421429. (doi:10.1111/ele.12086)

29. Gellner G, McCann KS, Hastings A. 2016 The duality of stability: towards a stochastic theory of species interactions. Theor. Ecol. 9, 477-485. (doi:10.1007/s12080-016-0303-2)

30. Hastings A et al. 2018 Transient phenomena in ecology. Science 361, eaat6412. (doi:10.1126/science.aat6412)

31. Morozov A et al. 2019 Long transients in ecology: Theory and applications. Phys. Life Rev. , S1571064519301447. (doi:10.1016/j.plrev.2019.09.004)

32. Pineda-Krch M, J. Blok H, Dieckmann U, Doebeli M. 2007 A tale of two cycles distinguishing quasi-cycles and limit cycles in finite predator-prey populations. Oikos 116, 53-64. (doi:10.1111/j.2006.0030-1299.14940.x)

33. Hastings A, Abbott KC, Cuddington K, Francis TB, Lai Y-C, Morozov A, Petrovskii S, Zeeman ML. 2021 Effects of stochasticity on the length and behaviour of ecological transients. J. R. Soc. Interface. 18, 20210257. (doi:10.1098/rsif.2021.0257)

34. Touboul J, Krupa M, Desroches M. 2015 Noise-induced canard and mixed-mode oscillations in large-scale stochastic networks. SIAM J. Appl. Math. 75, 2024-2049.

35. Poggiale J-C, Aldebert C, Girardot B, Kooi BW. 2020 Analysis of a predator-prey model with specific time scales: a geometrical approach proving the occurrence of canard solutions. J. Math. Biol. 80, 39-60. (doi:10.1007/s00285-019-01337-4) 
36. Wichmann MC, Johst K, Schwager M, Blasius B, Jeltsch F. 2005 Extinction risk, coloured noise and the scaling of variance. Theor. Pop. Biol. 68, 29-40.

(doi:10.1016/j.tpb.2005.03.001)

37. Bezanson J, Edelman A, Karpinski S, Shah VB. 2017 Julia: A fresh approach to numerical computing. SIAM Rev. 59, 65-98. (doi:10.1137/141000671)

38. Gounand I, Mouquet N, Canard E, Guichard F, Hauzy C, Gravel D, Connolly AESR, Bronstein EJL. 2014 The paradox of enrichment in metaecosystems. Am. Nat. 184, 752763. (doi:10.1086/678406)

39. Meyer K, Hoyer-Leitzel A, lams S, Klasky I, Lee V, Ligtenberg S, Bussmann E, Zeeman ML. 2018 Quantifying resilience to recurrent ecosystem disturbances using flow-kick dynamics. Nat Sustain 1, 671-678. (doi:10.1038/s41893-018-0168-z)

40. Higgins K, Hastings A, Sarvela JN, Botsford LW. 1997 Stochastic dynamics and deterministic skeletons: Population behavior of Dungeness Crab. Science 276, 14311435. (doi:10.1126/science.276.5317.1431)

41. Nolting BC, Abbott KC. 2016 Balls, cups, and quasi-potentials: quantifying stability in stochastic systems. Ecology 97, 850-564. (doi:10.1890/15-1047.1)

42. Jordano P. 2016 Chasing ecological interactions. PLOS Biol. 14, e1002559. (doi:10.1371/journal.pbio.1002559)

43. Lafferty KD, DeLeo G, Briggs CJ, Dobson AP, Gross T, Kuris AM. 2015 A general consumer-resource population model. Science 349, 854-857. 


\section{Supporting Information for "Slow organisms exhibit \\ 431 sudden population disappearances in a reddened world"}

\section{Authors:}

433 Christopher J. Greyson-Gaito ${ }^{* 1}$, Gabriel Gellner ${ }^{1}$, Kevin S. McCann ${ }^{1}$

\section{Affiliations:}

435 1. Department of Integrative Biology, University of Guelph, Guelph, Ontario, Canada

436 *Corresponding Author - Email: christopher@greyson-gaito.com (CJGG)

\section{ORCID:}

CJGG - 0000-0001-8716-0290

439 GG - 0000-0001-8170-1463

$440 \quad \mathrm{KSM}-000-0001-6031-7913$

\section{Proof of excitability decreasing when slowing the consumer}

442 When examining the effect of slowing the consumer (increasing $1 / \varepsilon$ ) in the local region around 443 the interior equilibrium using the standard linear perturbation technique, the excitability of the 444 dynamics decreases. We prove this below using the more tractable consumer-resource 445 model with type I functional response that has been non-dimensionalized.

446 With change of variables

$447 \quad X=x k, Y=\frac{y r}{a}, t=\frac{t}{r}$

448 and with non-dimensional parameters $\alpha=\frac{k a e}{r}, \beta=\frac{m}{r}$

449 the non-dimensionalized form of the type I functional response consumer-resource model is:

$450 \frac{d x}{d t}=x(1-x)-x y$ 
$451 \frac{d y}{d t}=\epsilon(\alpha x y-\beta y)$

452 Equilibria exist at

$453 \widehat{x}_{1}=0, \widehat{y}_{1}=0$

$454 \widehat{x}_{2}=\frac{\beta}{\alpha}, \widehat{y}_{2}=1-\frac{\beta}{\alpha}$

455 The jacobian of the model is

$456 \quad\left(\begin{array}{cc}1-2 x-y & -x \\ \varepsilon \alpha y & \varepsilon(\alpha x-\beta)\end{array}\right)$

457 Inputting the interior equilibrium, $\widehat{x}_{2}, \widehat{y}_{2}$, into the jacobian returns

$458\left(\begin{array}{cc}\frac{-\beta}{\alpha} & \frac{-\beta}{\alpha} \\ \varepsilon(\alpha-\beta) & 0\end{array}\right)$

459 Using the trace and determinant of this jacobian matrix we can get the characteristic 460 polynomial and the quadratic equation to solve for the eigenvalues:

$461 \quad$ Trace $=\frac{-\beta}{\alpha}$

462 Determinant $=\frac{\beta \varepsilon(\alpha-\beta)}{\alpha}$

463 Characteristic polynomial $=\lambda^{2}+\frac{\beta}{\alpha} \lambda+\frac{\beta \varepsilon(\alpha-\beta)}{\alpha}$

464

$$
\lambda=\frac{\frac{-\beta}{\alpha} \pm \sqrt{\left(\frac{\beta}{\alpha}\right)^{2}-4 \frac{\beta \varepsilon(\alpha-\beta)}{\alpha}}}{2}
$$

465 We are determining the boundary of real to complex eigenvalues, thus we must examine what 466 is inside the square root of the quadratic equation:

467 When $\left(\frac{\beta}{\alpha}\right)^{2}-4 \frac{\beta \varepsilon(\alpha-\beta)}{\alpha}<0$ the eigenvalues are complex 
468

469

470

471

472

473

474

475

476

We can solve for $\alpha$ to find what parameter values produce $\alpha$ at the real/complex divide

$$
a=\frac{\beta \epsilon \pm \sqrt{\beta \epsilon(\beta \epsilon+1)}}{2 \epsilon}
$$

We can ignore the minus square root part (because $\beta \epsilon<\sqrt{\beta \epsilon(\beta \epsilon+1)}$ always and we get a negative alpha value which is impossible biologically).

Thus, we concentrate on

$\alpha=\frac{\beta \epsilon+\sqrt{\beta \epsilon(\beta \epsilon+1)}}{2 \epsilon}$

We can differentiate the above equation with respect to $\varepsilon$ to find out how the a value (at which the real/complex divide occurs) changes.

$\frac{d \alpha}{d \epsilon}=\frac{-\beta}{4 \epsilon \sqrt{\beta \epsilon(\beta \epsilon+1)}}$

which is always negative when $\beta$ and $\varepsilon$ are positive (biologically they have to be).

Therefore, if we decrease $\varepsilon$ (slowing the consumer by increasing $1 / \varepsilon$ ), the $\alpha$ value - at which the real/complex divide occurs - increases. Converting $\alpha$ back into its original dimensional parameters, we see that if $k$ and $a$ are kept constant, $e$ must increase to increase the nondimensional $\alpha$ parameter.

\section{Explanation of quasi-canard finder algorithm}

The algorithm checks that the trajectory has the characteristics of a quasi-canard. Thus, the algorithm includes a return map at the maximum point of the resource isocline where canards and quasi-canards must pass through. The algorithm also includes boxes along the attracting and repelling manifolds (the right side of the resource isocline and the consumer axis respectively) through which a quasi-canard should pass. The quasi-canard passes through these checks in a particular order and so the algorithm ensures the order is correct. Below are the six steps that the quasi-canard finder algorithm goes through. The full code can be found in slowfast_canardfinder.jl of the Github repository.

1. The algorithm finds all the points in the time series where the next sequential point creates a vector that intersects with a line that sits at the Hopf bifurcation point on the Resource isocline (the maximum of the Resource isocline). The line has a length of $5 \%$ of the Hopf bifurcation point above and below the Hopf bifurcation point. If no points are found, the algorithm does step six. If points are found, the points are collated and passed to the next step. 
2. The algorithm then takes all of these points and moves along the time series after these points to identify the first point within a box that sits between the Hopf bifurcation point and where the Resource isocline intersects with the Consumer axis. The box has a width of 0.1 . If no points are found, the algorithm does step six. If points are found, the points are collated and passed to the next step.

3. The algorithm then takes all of these points and moves along the time series after these points to identify the first point within a box that sits between the 0 consumers and $80 \%$ of where the Resource isocline intersects with the Consumer axis. The box has a width of 0.1 . If no points are found, the algorithm does step six. If points are found, the points are collated and passed to the next step.

4. The algorithm then takes all of these points and moves along the time series after these points to identify the first point that sits close to the resource isocline. If no points are found, the algorithm does step six. If points are found, the points are collated and passed to the next step.

5. The algorithm then takes all of these points and repeats step 1 to ensure a full cycle of the quasi-canard. If the return map check is passed for the second time, the algorithm returns "quasi-canard", otherwise the algorithm does step six.

517 Note, the sensitivity of this algorithm to find quasi-canards can be changed by varying the top

518 of the box in step three (changing the percentage of where the Resource isocline intersects

519 with the Consumer axis). 


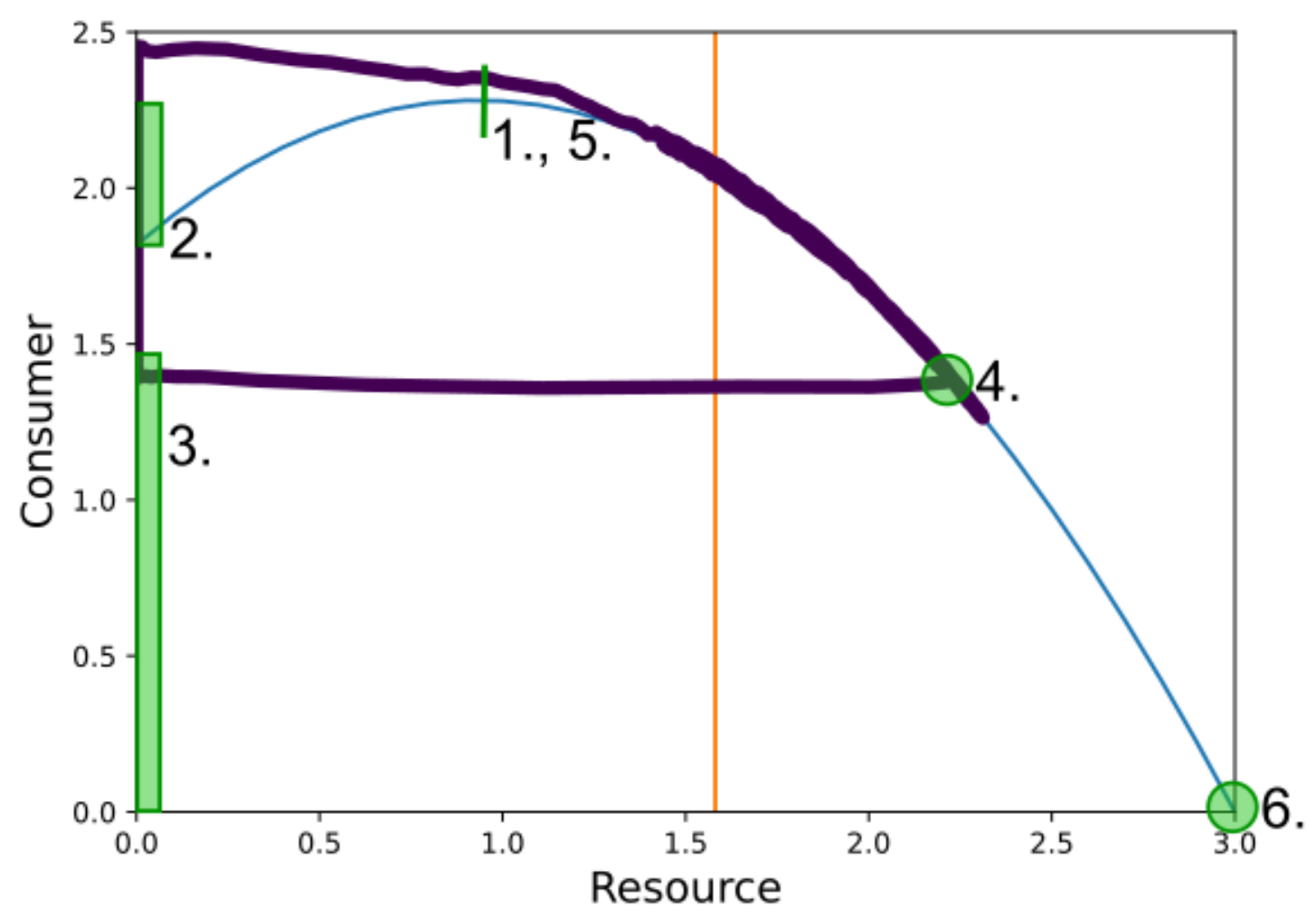

520 Figure 1 Consumer-Resource phase plot with a quasi-canard, the resource isocline and the 521 consumer isocline in purple, blue, and orange respectively. The six steps of the algorithm 522 outlined above are depicted with green lines, boxes, and circles. 
524 Isoclines of Rosenzweig-MacArthur consumer-resource model

525
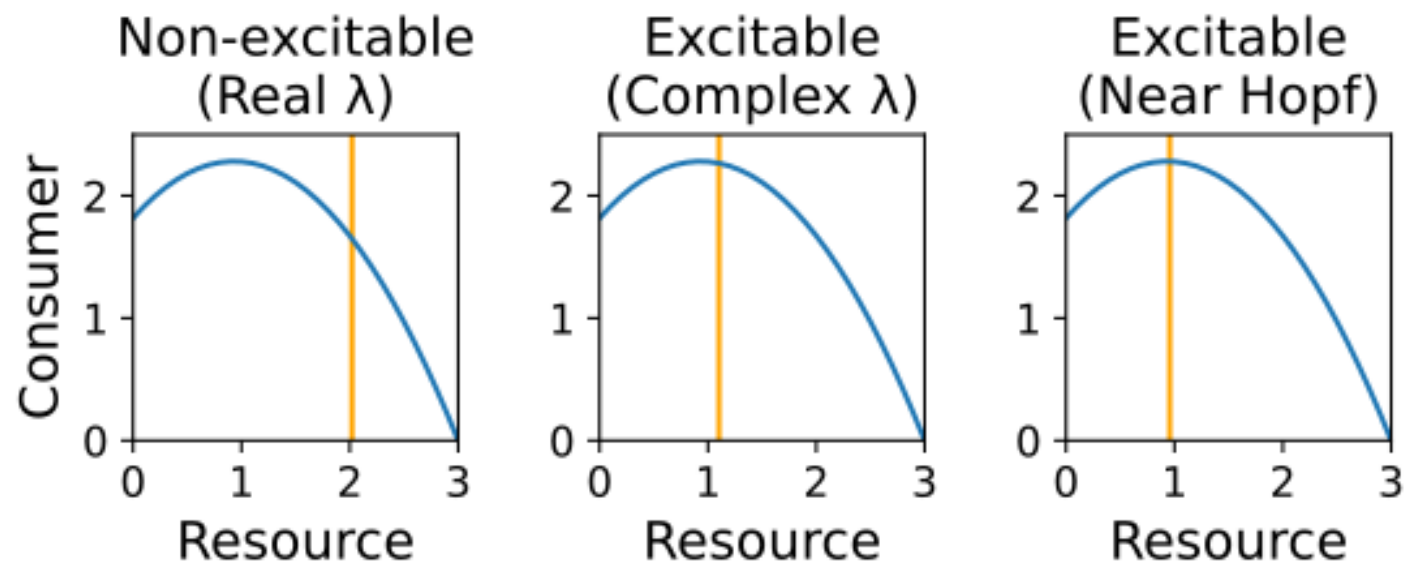

526 Figure 2 a), b), c) resource and consumer isoclines with efficiency values of $0.5,0.65,0.7$

527 respectively.

\section{Biologically Plausible Parameters}

529 We used Yodzis \& Innes' [26] biologically plausible parameterization of the consumer-

530 resource model to test whether our sudden population disappearance results are general to

531 other parameter sets.

$532 \frac{d R}{d t}=R\left(1-\frac{R}{K}\right)-\frac{\frac{x y}{(1-\delta) f_{e}} C R}{R+R_{0}}$

$533 \frac{d C}{d t}=C x\left(-1+\frac{y R}{R+R_{0}}\right)$

534 where $x=\left(\frac{a_{T}}{f_{r} a_{r}}\right)\left(\frac{m_{R}}{m_{C}}\right)^{0.25}$

$535 y=\frac{f_{J} a_{J}}{a_{T}}$

536 Similar to Yodzis \& Innes [26], we expressed the resource body mass in terms of the mass of

537 an equivalent endotherm operating at its physiological limit. We set our consumer as an

538 herbivorous endotherm.

539 Thus, $a_{T}=54.9, a_{r}=34.3, \delta=0.55, f_{J}=0.99, a_{J}=89.2, K=3.0$ 
540 To slow the consumer relative to the resource, we multiplied the resource/consumer body

541 mass ratio by $\varepsilon\left(\varepsilon \mathrm{B}=\varepsilon m_{E R} / m_{C}\right)$. To maintain the same biomass loss from the resource (i.e.

542 when using the original $\left.\mathrm{B}=m_{E R} / m_{C}\right)$, we set

$543 f_{e}=\frac{(\varepsilon B)^{0.25}}{B^{0.25}}$

544 To ensure the full model was feasible (between the feasibility and Hopf boundaries in Yodzis

$545 \&$ Innes [26]), we set $\mathrm{B}=10^{-6}$ and we restricted $R_{0}$ to $[0.7,1.82]$.

546 All other methods are the same as in the main Methods section.
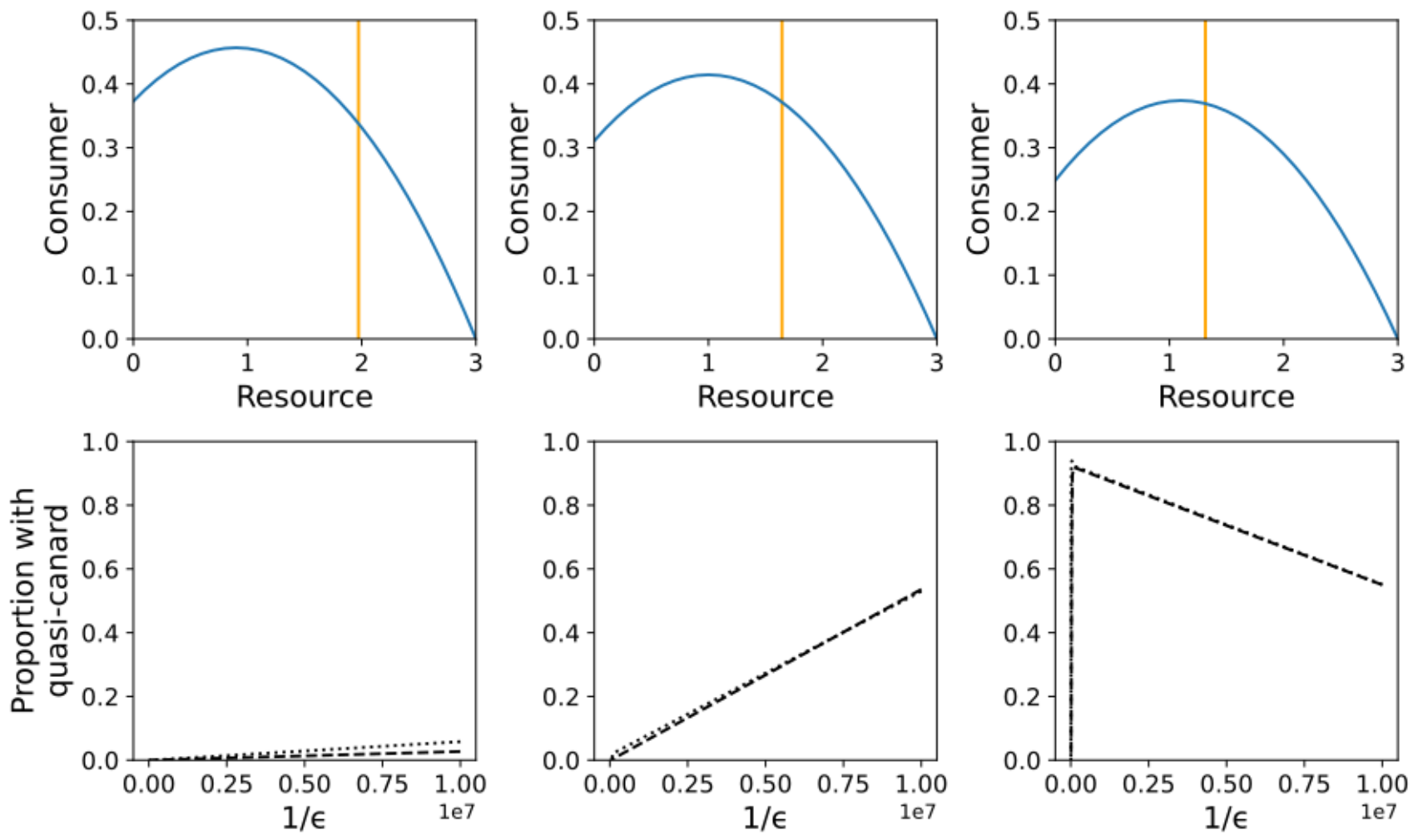

548 Figure 3 In the Yodzis \& Innes (1992) model, quasi-canards can be found for a variety of

549 efficiency values within certain boundaries of $\varepsilon$ parameter space. a), b), c), d) resource and

550 consumer isoclines with $R_{0}$ values of $1.2,1.0,0.8$ respectively. e), f), g), h) proportion of 1000

551 simulations per value of $1 / \varepsilon$ that exhibited quasi-canards with constant $R_{0}$ values of 1.2, 1.0,

5520.8 respectively. Bold and thin dashed lines correspond to 6,000 and 24,000 time steps

553 respectively. 


\section{Quasipotentials}

555 The quasipotentials depicted below were created using the Rosenzweig-MacArthur consumer 556 -resource model with the same parameter values as the model in the main article. We used 557 the QPot package (version 1.2) in R to calculate the quasipotentials (Moore et al. 2016). We 558 maintained the overall intensity of noise but had different relative noise intensities between 559 the resource and the consumer (specifically 1:4, see Moore et al. (2016) for how to specify 560 different relative noise intensities).

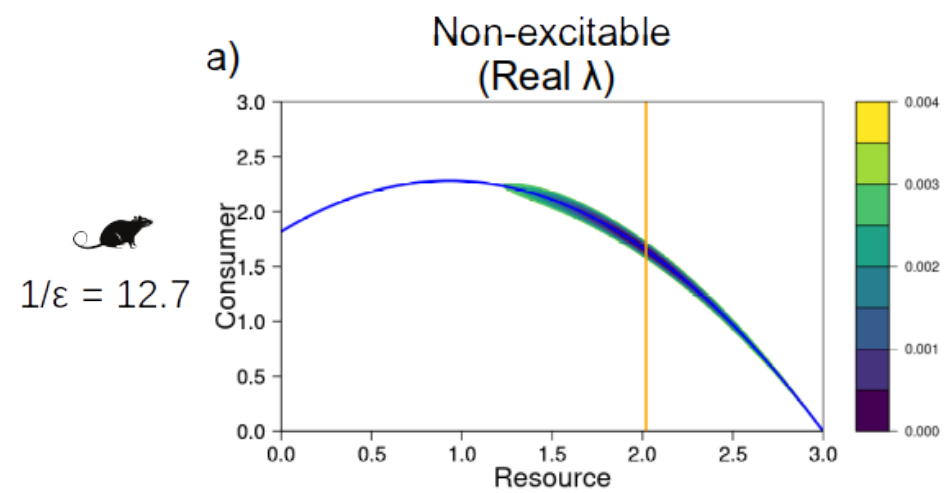

c)
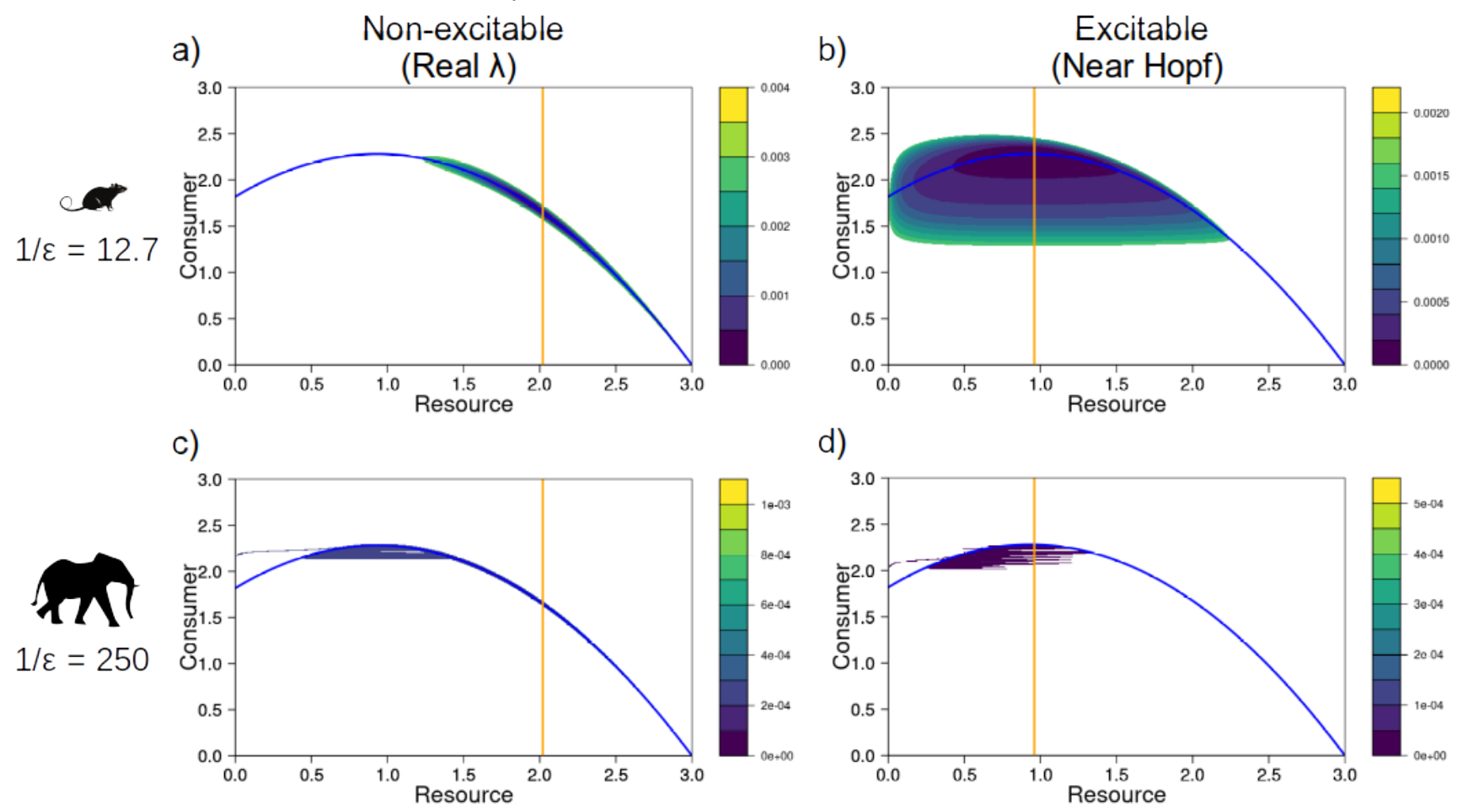

d)

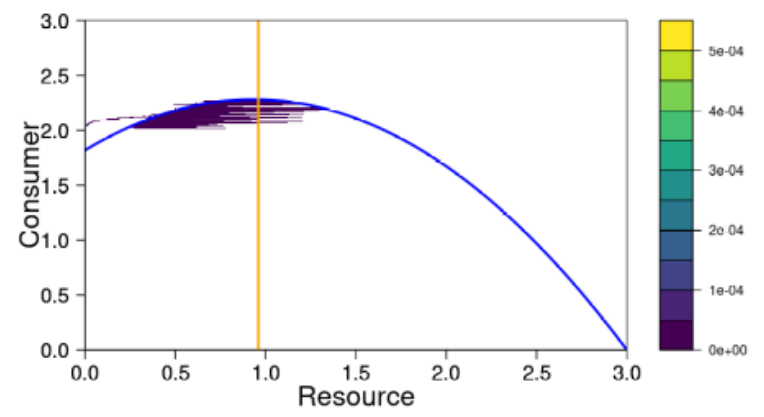

561 Figure 4 Quasipotentials for the consumer-resource models. The top row (a) \& b) ) had a $1 / \varepsilon$ value of 12.7 and the bottom row (c) \& d) ) had a $1 / \varepsilon$ value of 250 . Each column of plots had efficiency values of 0.5 and 0.7 respectively. Resource and consumer isoclines are the blue and orange lines respectively.

565 Christopher Moore, Christopher Stieha, Ben Nolting, Maria Cameron and Karen Abbott 566 (2016). QPot: Quasi-Potential Analysis for Stochastic Differential Equations. R package 567 version 1.2. https://github.com/bmarkslash7/QPot. 